\title{
Pragmatic Aspect of Translation: The Interpretation-Based Inference and Its Implications for Translation
}

\author{
Rafat Y. Alwazna \\ Department of European Languages and Literature \\ King Abdulaziz University \\ Jeddah, Kingdom of Saudi Arabia
}

\begin{abstract}
The elements of encoding, transferring and decoding are crucial in all processes of communication, however, drawing the appropriate inference from the current context is equally important in communication according to relevance theory (Gutt, 1998, p. 41). Semantic content is not always sufficient to fully comprehend the exact meaning of a particular utterance as the meaning of that utterance may hinge upon the contextual detail with which it is inferentially associated. The success of the process of communication relies on whether or not the recipient makes use of the context intended by the speaker. Failure to do so would give rise to miscommunication (Gutt, 1998, p. 42). Translation, as a communicative act, involves interpretation made by the translator, which takes the context of the target text (TT) reader and his/her knowledge into consideration. The present paper argues that even though the translator, according to relevance theory, is required to reproduce a TT that can stand as a faithful rendering of the source text (ST), the translator, however, needs to make his/her translated text relevant to the target reader. This, in many instances, may demand following certain procedures of explications in the TT to equip the target reader with the relevant contextual information needed to draw the appropriate inferences from the utterance concerned, and therefore make the right interpretation. Such exegesis needs to be added to the target text as what is inferable for the ST user may not be inferable for the TT receiver owing to cognitive and cultural differences.
\end{abstract}

Keywords: Communication, inference, interpretation, relevance theory, translation

Cite as: Alwazna, R. Y. (2017). Pragmatic Aspect of Translation: The Interpretation-Based Inference and Its Implications for Translation. Arab World English Journal for Translation \& Literary Studies, 1(4). DOI:http://dx.doi.org/10.24093/awejtls/vol1no4.3 